\title{
Determination of Analytical Solution and Limit Cycle of Nonlinear Chemical Oscillations
}

\author{
Dagbégnon Luc Olabodé ${ }^{1}$, Julien Yovogan ${ }^{2}, \&$ Jean Bio Chabi Orou ${ }^{1}$
}

${ }^{1}$ Laboratoire de Mécanique des Fluides, de la Dynamique Nonlinéaire et de la Modélisation des Systèmes Biologiques (LMFDNMSB); Institut de Mathématiques et de Sciences Physiques, Porto-Novo, Bénin

${ }^{2}$ Université Nationale des Sciences Technologiques, Ingénierie et mathématiques (UNSTIM), Abomey, Bénin

Correspondence: Dagbégnon Luc Olabodé, Institut de Mathématiques et de Sciences-Physiques, BP: 613 Porto-Novo, Bénin. E-mail: olabodeluc@gmail.com

Received: February 22, 2019

Accepted: March 16, 2019

Online Published: March 30, 2019

doi:10.5539/apr.v11n2p79

URL: https://doi.org/10.5539/apr.v11n2p79

\begin{abstract}
:
This work studies the determination of analytical solution and cycle limite in nonlinear chemical oscillations gouverned by a forced modified Van der Pol-Duffing oscillator. We considered the dynamic of nonlinear chemical systems subjected to an external sinusoidal excitation. The first order appoximative solution of the oxcillator is determined using the Lindstedt's perturbation method. The limit cycle number of the oscillator is found as well as the effect of certain parameters of the model on cycle limit analyzes. The harmonic balance method is used to find the amplitudes of the oscillatory states. The effect of the constraint parameter $\beta$ of the oscillator are observed on amplitude-response curves. Numerical simulations are used to validate the results obtained by analytical methods.
\end{abstract}

Keywords: Modified Van der Pol-Duffing oscillator, chemical dynamics, Self-sustained oscillator, Limit cycle.

\section{Introduction}

Over the last three decades, the interdisciplinary field of nonlinear dynamics has made enormous progress. The study of nonlinear oscillations is of great interest in several scientific fields (Boissonade \& De Kepper, 1980; Epstein \& Showalter, 1996). In nonlinear chemical dynamics, she has proven that some chemical oscillations can be modeled by oscillators. The search for the analytical solution of the equations and dynamic phenomena of these oscillators is sometimes complicated given their complexity. In this paper, the determination of the analytical solution and the limit cycle in some chemical oscillations modeled by the modified Van Der Pol-Duffing oscillator is performed using appropriate methods. We analyze the effects of the different parameters of this oscillator on the limit cycle. Subsequently, the effects on certain dynamic phenomena of the parameter $\beta$ which marks the difference between this oscillator and the classic Van Der Pol-Duffing oscillator is also analyzed.

The organization of the paper is as follows : The mathematical modeling of nonlinear chemical dynamics influenced by external periodic excitation forces is given in Section 2. Section 3 presents the analytical solution, the limit cycle of the modified Van Der Pol-Duffing oscillator as well as the effect of the parameter $\beta$ on certain dynamic phenomena of the oscillator. The conclusion is given in the last section.

\section{Model and equation of oscillations}

This work takes into account all nonlinear chemical systems as a kinetic example which can be described by the following equations (Boissonade, J and al.,1980, Epstein, I R and al.,1996, \& Olabodé, D. L. and al., 2018)

$$
\begin{aligned}
& A \stackrel{k_{1}}{\longrightarrow} X, \\
& B+X \stackrel{k_{2}}{\longrightarrow} 2 X, \\
& D+X \stackrel{k_{3}}{\longrightarrow} \text { products, } \\
& X \stackrel{k_{4}}{\longrightarrow} X^{\prime}, \\
& B+X^{\prime} \stackrel{k_{5}}{\longrightarrow} Y, \\
& Y \stackrel{k_{6}}{\longrightarrow} X^{\prime}+\text { products. }
\end{aligned}
$$

Based upon the laws of mass action and conservation and assuming that the sink of the product is a first order reaction, the self-oscillations in some nonlinear chemical systems can be modelised by the following single second order differential 
equation (Olabodé, Miwadinou, Monwanou, \& Chabi Orou, 2018; Miwadinou et al., 2018)

$$
\ddot{\zeta}+\mu\left(1-\zeta^{2}\right) \dot{\zeta}+\alpha \zeta+\gamma \zeta^{3}+\beta=0
$$

where $\zeta$ is proportional to the concentration of species $X$ and $\mu, \alpha, \gamma, \beta$ respectively denote the damping coefficient, linear and cubic nonlinear restoring parameters. In general if $\alpha<0$ the system has three steady states and single one for $\alpha>0$. In the rest of this work, we consider the case of $\alpha>0$. Previous research has shown the existence of a richness of complex dynamics behaviors can be obtained when dissipative self-oscillators are submitted to external forcing. By assuming that the model is subjected to an external sinusoidal excitation $E \cos \Omega t$, Eq. (7) becomes a nonlinear single second order differential equation on the form

$$
\ddot{\zeta}+\mu\left(1-\zeta^{2}\right) \dot{\zeta}+\alpha \zeta+\gamma \zeta^{3}+\beta=E \cos \Omega t .
$$

where $\zeta, \dot{\zeta}$ and $\ddot{\zeta}$ are the displacement, velocity and acceleration respectively. $F$ and $\Omega$ are respectively the amplitude and the frequence of the excitation.

\section{Analytical solution and limit cycle}

\subsection{Amplitudes and frequencies of limit-cycles in autonomous chemical oscillations}

In this subsection, we consider the case where the model is not influenced by an external excitation $(E=0)$ and our purpose is to find the amplitudes and frequencies of the limit cycles.Using the Lindsted perturbation method (Lam, 1997; Rand, 2005), it is interesting to set $\tau=\omega t$ where $\omega$ is an unknown frequency, to allow an interaction between the frequency and the amplitude.The approximate periodic solution $\zeta(\tau)$ of equation (8) without external force can be written as follows:

$$
\zeta(\tau)=\zeta_{0}(\tau)+\mu \zeta_{1}(\tau)+\mu^{2} \zeta_{2}(\tau)+\ldots
$$

where the functions $\zeta_{j}(j=0,1,2, \ldots)$ are periodic functions of $\tau_{1}$ of period $2 \pi$.Furthermore, the frequency $\omega$ can be defined as follows:

$$
\omega=\omega_{0}+\mu \omega_{1}+\mu^{2} \omega_{2}+\ldots
$$

where the frequencies $\omega_{j}$ are unknown constants at this point. We put $\gamma=\mu \gamma_{0}$ and $\beta=\mu \beta_{0}$. By introducing the expression$\mathrm{s}(9)$ and (10) in Eq.(8) and equating the coefficients of $\mu^{0}, \mu^{1}$ and $\mu^{2}$ to zero, we find the following equations at different orders of $\mu$ :

Order $\mu^{0}$

$$
\omega_{0}^{2} \ddot{\zeta}_{0}+\alpha \zeta_{0}=0
$$

Order $\mu^{1}$

$$
\omega_{0}^{2} \ddot{\zeta}_{1}+\alpha \zeta_{1}=-2 \omega_{0} \omega_{1} \ddot{\zeta}_{0}-\omega_{0}\left(1-\zeta_{0}^{2}\right) \dot{\zeta}_{0}-\gamma_{0} \zeta_{0}^{3}-\beta_{0}
$$

Order $\mu^{2}$

$$
\omega_{0}^{2} \ddot{\zeta}_{2}+\alpha \zeta_{2}=-\left(\omega_{1}^{2}+2 \omega_{0} \omega_{2}\right) \ddot{\zeta}_{0}-2 \omega_{0} \omega_{1} \ddot{\zeta}_{1}-\omega_{1}\left(1-\zeta_{0}^{2}\right) \dot{\zeta}_{0}-\omega_{0}\left(1-\zeta_{0}^{2}\right) \dot{\zeta}_{1}+2 \omega_{0} \zeta_{0} \zeta_{1} \dot{\zeta}_{0}-3 \gamma_{0} \zeta_{0}^{2} \zeta_{1}
$$

The unknown quantities in the above equations are determined using the following characteristics of functions $\zeta_{j}$ :

$$
\zeta_{j}(\tau+2 \pi)=\zeta_{j}(\tau) \quad \text { and } \quad \dot{\zeta}_{j}(0)=0 ; \quad j=0,1,2
$$

After solving Eq.(11) and using conditions (14), we have

$$
\zeta_{0}=A \cos (\sqrt{\alpha} \tau)
$$

$$
\omega_{0}=\sqrt{\alpha}
$$

where $A$ is the amplitude of the limit cycle. The introduction of the solution Eq. (15) and the relation (16) in Eq. (12) leads to: 


$$
\ddot{\zeta}_{1}+\zeta_{1}=\left(2 \omega_{1} A \sqrt{\alpha}-\frac{3 \gamma_{0} A^{3}}{4 \alpha}\right) \cos (\sqrt{\alpha} \tau)+\left(1-\frac{A^{2}}{4}\right) A \sin (\sqrt{\alpha} \tau)-\frac{\gamma_{0} A^{3}}{4 \alpha} \cos (3 \sqrt{\alpha} \tau)-\frac{A^{3}}{4} \sin (3 \sqrt{\alpha} \tau)-\frac{\beta_{0}}{\alpha}
$$

The secularity conditions on this last equation give the following equation satisfies by the amplitude $A$ of the limit cycle:

$$
1-\frac{A^{2}}{4}=0
$$

and

$$
\omega_{1}=\frac{3 \gamma_{0} A^{2}}{8 \alpha \sqrt{\alpha}}
$$

Thus, a general expression for a periodic solution of Eq.(17) can be written as follows:

$$
\zeta_{1}=A_{1} \cos (\sqrt{\alpha} \tau)+A_{2} \sin (\sqrt{\alpha} \tau)+C_{1} \cos (3 \sqrt{\alpha} \tau)+C_{2} \sin (3 \sqrt{\alpha} \tau)+C
$$

with

$$
\begin{aligned}
& A_{1}=0, \\
& A_{2}=\frac{A\left(4-A^{2}\right)}{4(1-\alpha)}, \\
& C_{1}=\frac{\gamma_{0} A^{3}}{4 \alpha(9 \alpha-1)}, \\
& C_{2}=\frac{A^{3}}{4(9 \alpha-1)}, \\
& C=-\frac{\beta_{0}}{\alpha}
\end{aligned}
$$

Finaly,

$$
\zeta_{1}=\frac{A\left(4-A^{2}\right)}{4(1-\alpha)} \sin (\sqrt{\alpha} \tau)+\frac{\gamma_{0} A^{3}}{4 \alpha(9 \alpha-1)} \cos (3 \sqrt{\alpha} \tau)+\frac{A^{3}}{4(9 \alpha-1)} \sin (3 \sqrt{\alpha} \tau)-\frac{\beta_{0}}{\alpha}
$$

The secularity conditions on equation Eq.(13) after the introduction of the solutions Eq. (15), Eq. (22) and the relations (16), (19) in equation leads to:

$$
\begin{gathered}
\omega_{2}=-\frac{9 \gamma_{0}^{2} A^{4}}{128 \alpha^{3} \sqrt{\alpha}}-\frac{\left(4-A^{2}\right)^{2}}{32(\alpha-1) \sqrt{\alpha}}-\frac{A^{4}}{32(9 \alpha-1) \sqrt{\alpha}}+\frac{3 \gamma_{0}^{2} A^{4}}{32 \alpha^{2}(9 \alpha-1) \sqrt{\alpha}} . \\
\frac{35 \alpha-3}{12(9 \alpha-1)} A^{2}-1=0
\end{gathered}
$$

So that, the solution of Eq.(7) without the external excitation can be approximated by

$$
\zeta(t)=-\frac{\beta}{\alpha}+A \cos (\omega \sqrt{\alpha} t)+\frac{\gamma A^{3}}{4 \alpha(9 \alpha-1)} \cos (3 \omega \sqrt{\alpha} t)+\mu\left\{\frac{A\left(4-A^{2}\right)}{4(1-\alpha)} \sin (\omega \sqrt{\alpha} t)+\frac{A^{3}}{4(9 \alpha-1)} \sin (3 \omega \sqrt{\alpha} t)\right\}+O\left(\mu^{2}\right)
$$

where the frequency $\omega$ is given by

$$
\omega=\sqrt{\alpha}+\frac{3 \gamma A^{2}}{8 \alpha \sqrt{\alpha}}+\mu^{2} \omega_{2}+O\left(\mu^{3}\right)
$$

The resolution of Eq.(18) gives a single possible value for the amplitude of the limit cycle $(A=2)$. We therefore note that the chemical model considered presents in its dynamics a single limit cycle. The limit cycle of the model is illustrated using the analytical solution given by Eq.(25) and a direct numerical simulation of Eq.(7) (see Fig.1). The evolution of the solution $\zeta(t)$ when the time $t$ varies is also illustrated (see Fig.2). It is noticed a good agreement for the comparison between the curve obtained analytically and that obtained by numerical simulation. We therefore retain that the analytical solution found for Eq.(8) without external force approaches the exact solution of this equation very well and that the limit 


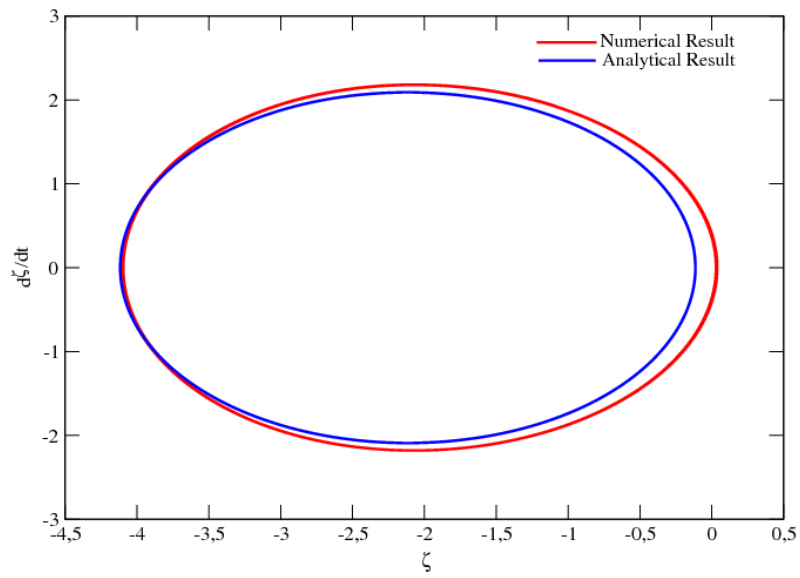

Figure 1: Phase portraits of the one limit cycle with the parameters $\mu=0.0001 ; \alpha=1.04 ; \gamma=0.005 ; \beta=2.2$ and $A=2$.

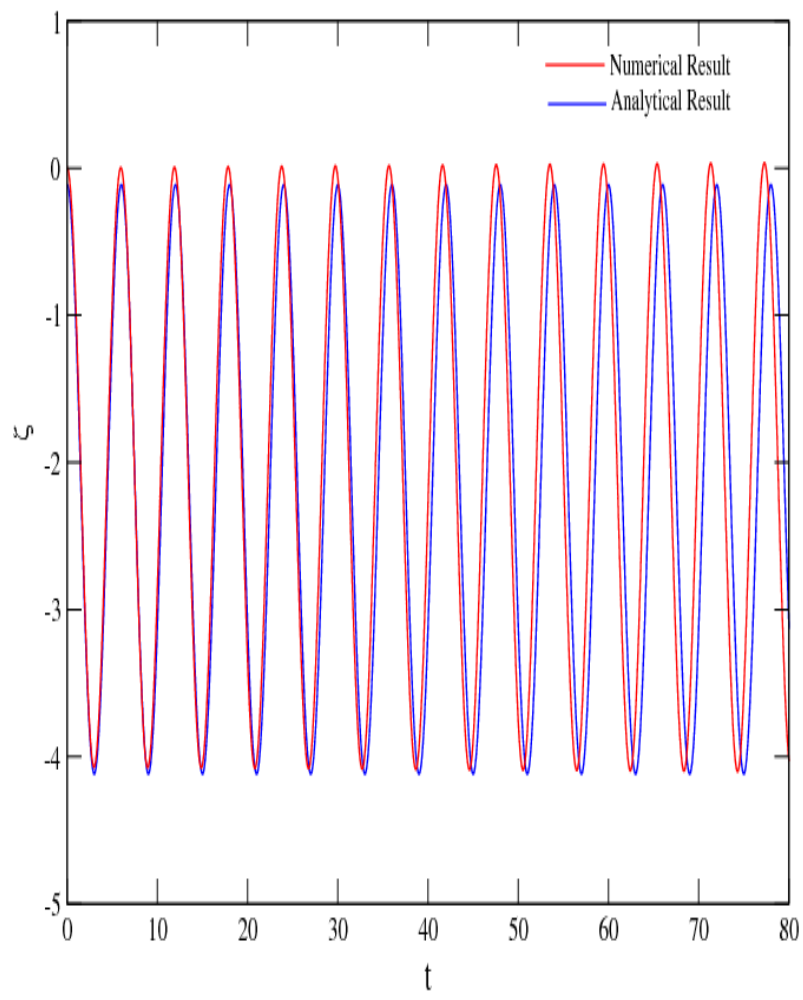

Figure 2: Time Historis with the parameters $\mu=0.0001 ; \alpha=1.04 ; \gamma=0.005 ; \beta=2.2$ and $A=2$. 

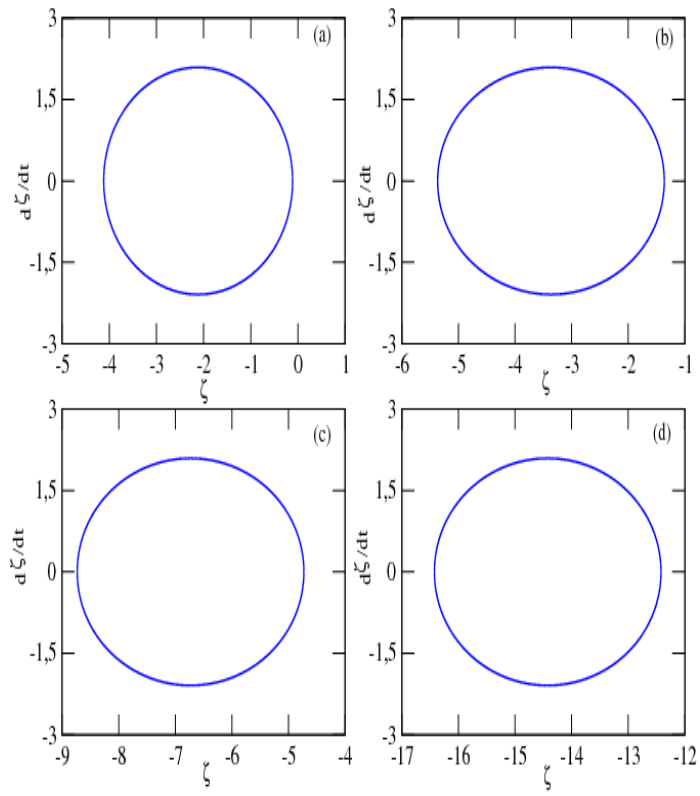

Figure 3: Effect of the parameter $\beta$ on the limit cycle for $(a) \beta=2.2,(b) \beta=3.50,(c) \beta=7,(d) \beta=15$ with the parameters of Fig1.
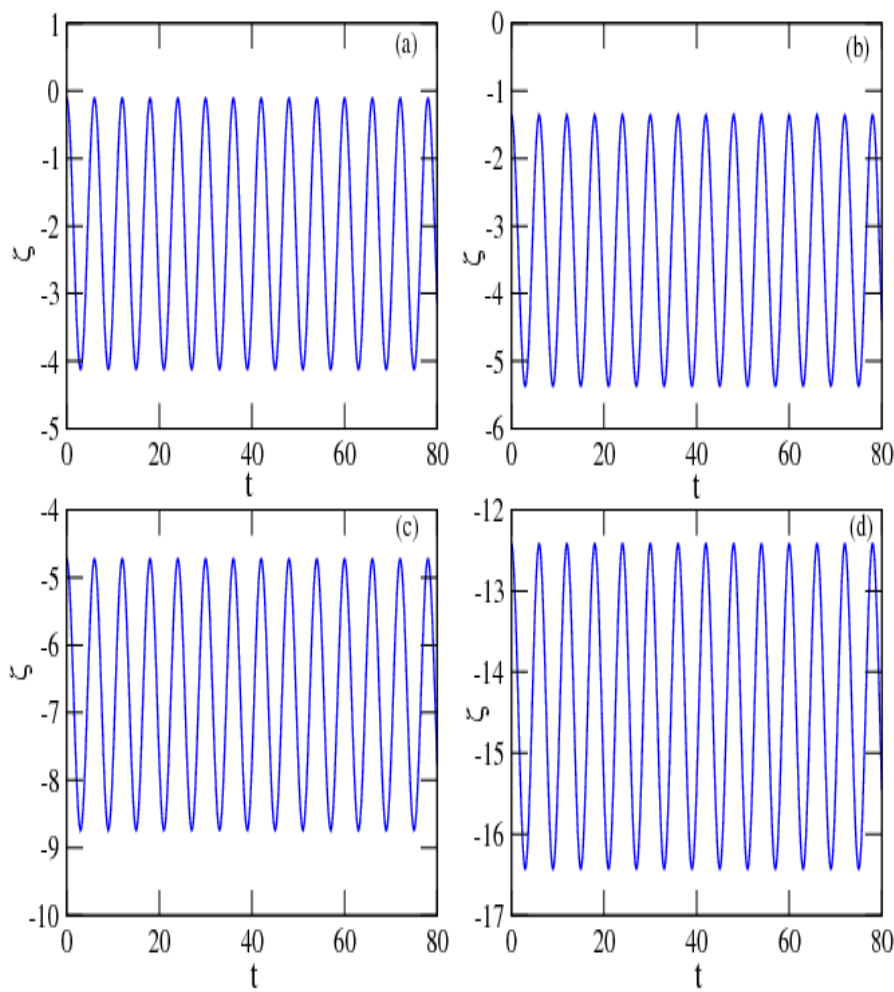

Figure 4: Effect of the parameter $\beta$ on Time Historis for $(a) \beta=2.2,(b) \beta=3.5,(c) \beta=7,(d) \beta=15$ with the parameters of Fig1. 

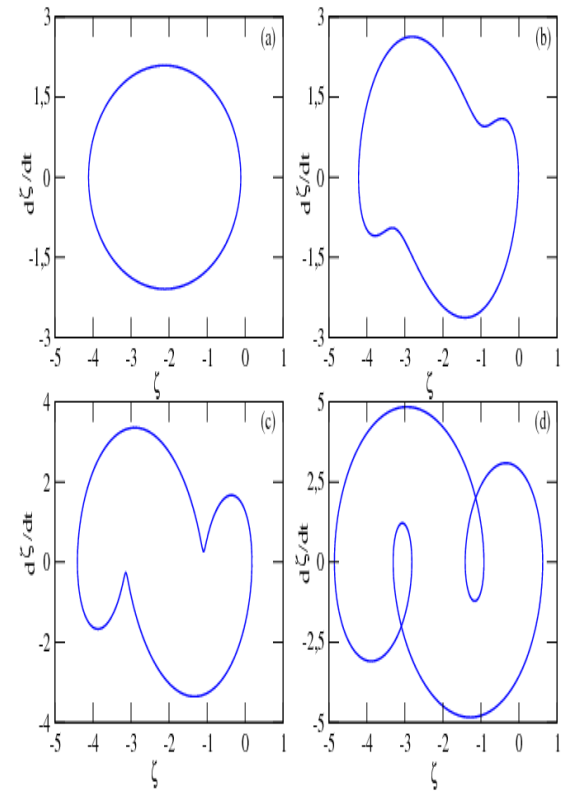

Figure 5: Effect of the parameter $\mu$ on the limit cycle for $(a) \mu=0.0001,(b) \mu=1,(c) \mu=2,(d) \mu=4$ with the parameters of Fig1.

cycle obtained is stable. The effects of certain parameters of the model are observed on the limit cycle. The influence of the parameter $\beta$ is observed on the phase space of the model (see Fig.3) and on his Time Historis (see Fig.4). We note a displacement of the limit cycle towards the abscissa $\zeta$ very weak and the stability of the limit cycle not changed when $\beta$ increases. The influence of the parameter $\mu$ on the limit cycle is illustrated in Fig.5 and we observe a tripling of the period of the limit cycle when the parameter $\mu$ increases. In the case where the model is influenced by the external excitation $(E \neq 0)$ many phenomena and behaviors can be hoping.

\subsection{Amplitude of the harmonic oscillatory states}

When the model is now influenced by external excitation, the amplitude of the forced harmonic oscillatory states can be found using the harmonic balance method (Nayfey, A. H. \& Mook, D. T., 1979). Our objective is to study the interaction between the external excitation and the amplitude of the limit cycle. For this reason, we assume that the fundamental component of the solutions has the period of external excitation and thus express the solution $\zeta$ as follows:

$$
\zeta=a_{1} \cos \Omega t+a_{2} \sin \Omega t-\frac{\beta}{\alpha}=A_{n c} \cos (\Omega t-\phi)-\frac{\beta}{\alpha} .
$$

By introducing expression (27) in Eq.(8) and by separately equalizing the coefficient of terms in sine and cosine, we obtain

$$
\begin{aligned}
& \left(\alpha-\Omega^{2}+3 \gamma \frac{\beta^{2}}{\alpha^{2}}+\frac{3}{4} \gamma A_{n c}^{2}\right) a_{1}-\mu \Omega\left(\frac{\beta^{2}}{\alpha^{2}}-1+\frac{1}{4} A_{n c}^{2}\right) a_{2}=E \\
& \mu \Omega\left(\frac{\beta^{2}}{\alpha^{2}}-1+\frac{1}{4} A_{n c}^{2}\right) a_{1}+\left(\alpha-\Omega^{2}+3 \gamma \frac{\beta^{2}}{\alpha^{2}}+\frac{3}{4} \gamma A_{n c}^{2}\right) a_{2}=0
\end{aligned}
$$

where

$$
A_{n c}^{2}=a_{1}^{2}+a_{2}^{2}, \quad \tan \phi=\frac{\mu \Omega\left(\frac{\beta^{2}}{\alpha^{2}}-1+\frac{1}{4} A_{n c}^{2}\right)}{\left(\alpha-\Omega^{2}+3 \gamma \frac{\beta^{2}}{\alpha^{2}}+\frac{3}{4} \gamma A_{n c}^{2}\right)} .
$$

The following nonlinear algebraic equation satisfied by the amplitude $A_{n c}$ is obtained after some algebraic manipulations of equation (28):

$$
\begin{aligned}
& \frac{1}{16}\left(\frac{9 \gamma^{2}}{\mu^{2} \Omega^{2}}+1\right) A_{n c}^{6}+\frac{1}{2}\left[\frac{3 \gamma\left(\alpha-\Omega^{2}\right) \alpha^{2}+9 \gamma^{2} \beta^{2}}{\mu^{2} \Omega^{2} \alpha^{2}}+\frac{\beta^{2}}{\alpha^{2}}-1\right] A_{n c}^{4}+\left[\left(\frac{\beta^{2}}{\alpha^{2}}-1\right)^{2}+\frac{1}{\mu^{2} \Omega^{2}}\left(\alpha-\Omega^{2}+\frac{3 \gamma \beta^{2}}{\alpha^{2}}\right)^{2}\right] A_{n c}^{2}- \\
& \frac{E^{2}}{\mu^{2} \Omega^{2}}=0
\end{aligned}
$$




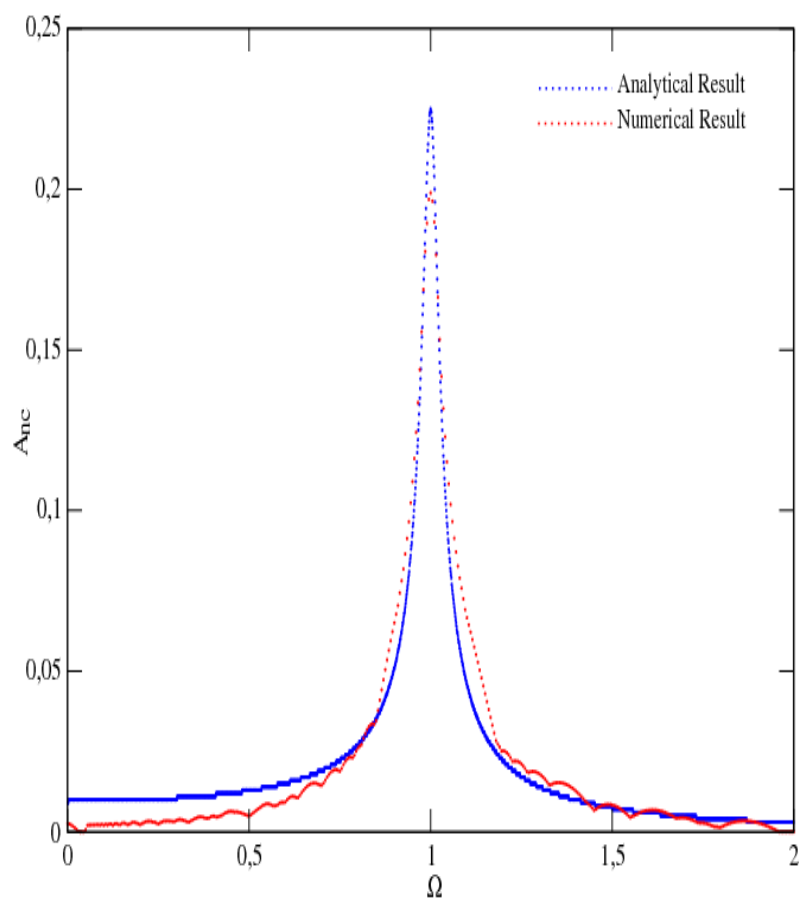

Figure 6: Comparison between analytical and numerical frequency-response curve $A_{n c}(\Omega)$ with the parameters $\mu=0.045$; $\alpha=1 ; \gamma=0.02 ; \beta=0.008$ and $E=0.01$.

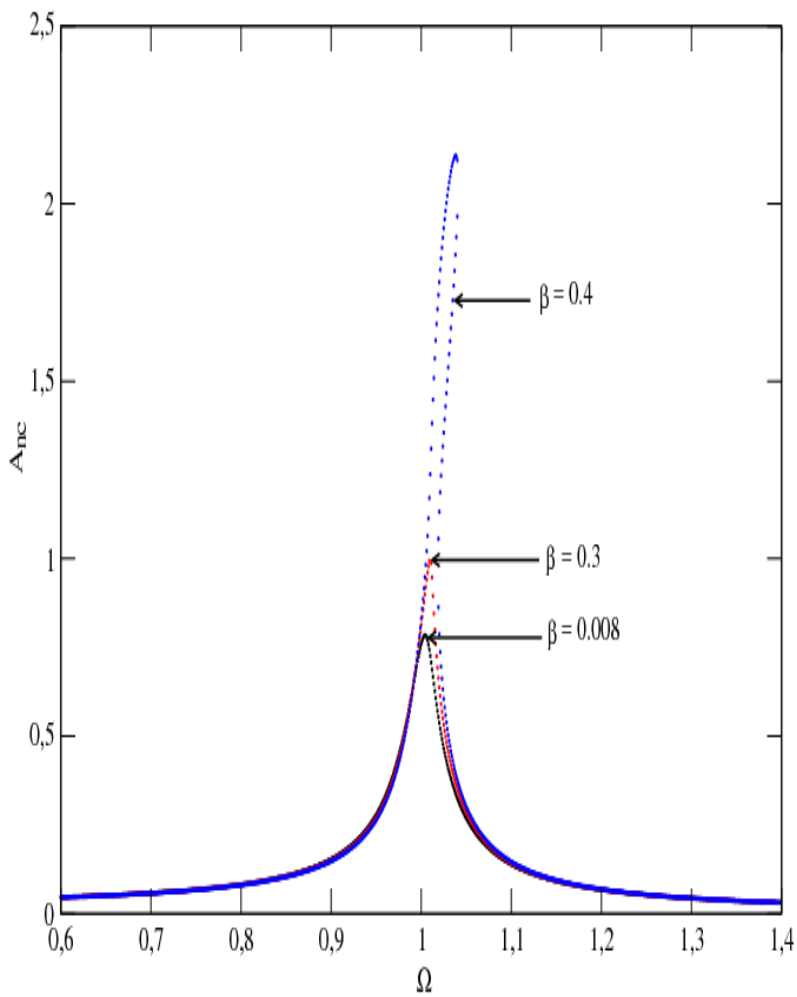

Figure 7: Effects of the parameter $\beta$ on the amplitude of the harmonic oscillatory states $A_{n c}(\Omega)$ with the parameters $\mu=0.045 ; \alpha=1 ; \gamma=0.02$ and $E=0.03$. 


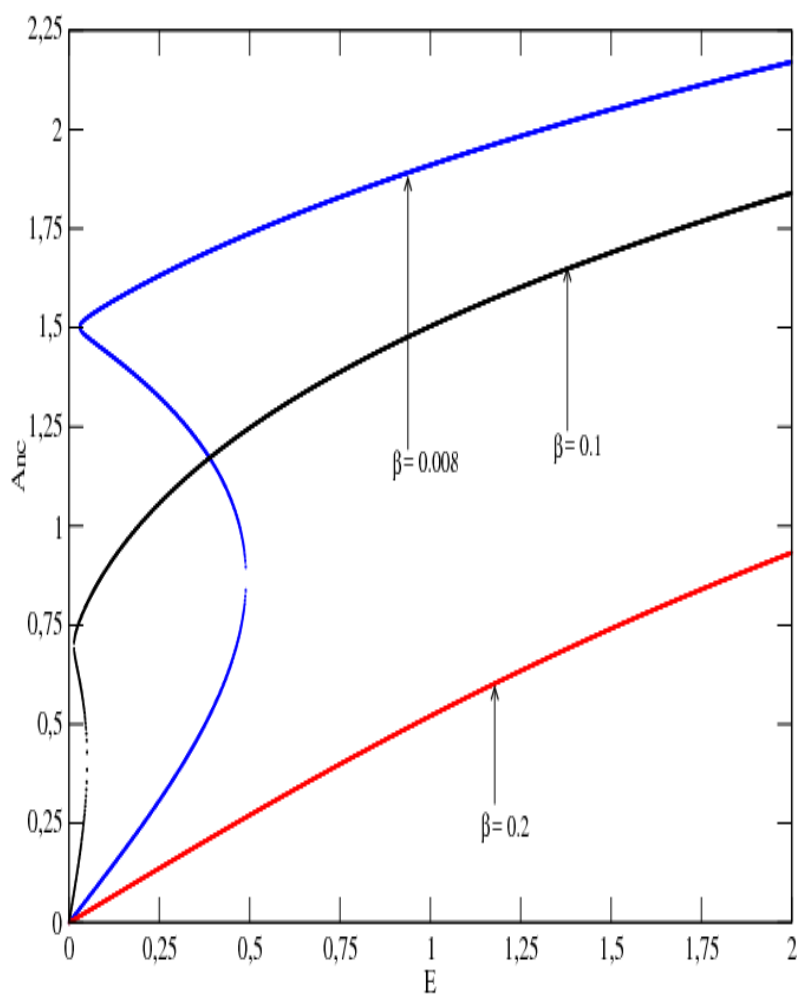

Figure 8: Effects of the parameter $\beta$ on the amplitude-response curve displaying jump in amplitude $A_{n c}(E)$ with the parameters $\mu=0.045 ; \alpha=0.15 ; \gamma=0.5$ and $\Omega=1$.

Equation (30) is solved using the Newton Raphson algorithm and the behavior of the amplitude $A_{n c}$ is observed when the frequency of the external excitation $\Omega$ is varied. We obtained a good agreement for the comparison between the analytical frequency response curve obtained via Eq.(30) and that obtained from the direct numerical simulation of Eq.(8) (see Fig.6). We have investigated later the effect of the parameter $\beta$ on the state of resonance, the process of hysteresis and the amplitude jump and the results are illustrated by the figures 7,8. The fig.7 shows the variation of $A_{n c}$ as a function of $\Omega$ for different values of $\beta$. It is noted through this figure that the amplitude of the resonance and the phenomenon of amplitude jump increases with $\beta$. The fig.8 shows the variation of $A_{n c}$ as a function of the amplitude $E$ of the external excitation. This figure shows that the phenomenon of hysteresis disappears with the increase of the parameter $\beta$. We can therefore conclude that the parameter $\beta$ which shows the difference between this modified Van der Pol-Duffing oscillator and the ordinary Van der Pol-Duffing oscillator already studied by many researchers in the literature, can be used to reduce or suppress the amplitude of the resonance and the hysteresis phenomenon in the dynamics of the nonlinear chemical reactions considered.

\section{Conclusion}

In this paper we have investigated on the determination of analytical solution and cycle limite in nonlinear chemical dynamics. We have considered chemical dynamics modeled by a modified Van der Pol-Duffing oscillator subjected to external periodic excitation. The model has been described and the corresponding equation of motion obtained.The Lindsted perturbation method is used to determine the amplitude and frequency of the model boundary cycle as well as an approximate solution of the oscillator. The amplitude of the oscillatory states in the non-autonomous case was found using the method of the harmonic balance. In the dynamics of the model we noticed the appearance of some very important phenomena such as resonance, hysteresis and amplitude jump. The influence of certain parameters of the model on these phenomena has been studied.

\section{Acknowledgements}

The authors thank IMSP-UAC and DAAD for financial support. We would also like to thank in a specialway the anonymous referees whose useful critics, comments and suggestions would helped strengthen the content and the quality of the paper. 


\section{References}

Boissonade, J., \& De Kepper, P. (1980). Transitions from Bistability to Limit Cycle Oscillations. Theoretical Analysis and Experimental Evidence in an Open Chemical System. J. Phys. Chem., 84, 501-506 .

Epstein, I. R., \& Showalter, K. (1996). Nonlinear Chemical Dynamics: Oscillations, Patterns, and Chaos. J. Phys. Chem., 100, 13132-13147.

Olabodé, D. L., Miwadinou, C. H., Monwanou, A. V., \& Chabi Orou, J. B. (2018). Horseshoes chaos and its passive control in dissipative nonlinear chemical dynamics. Physica Scripta, 93, 085203

Miwadinou, C. H., Monwanou, A. V., Yovogan, J., Hinvi, L. A., Nwagoum Tuwa, P. R., \& Chabi Orou, J. B. (2018). Modeling nonlinear dissipative chemical dynamics by a forced modifed Van der Pol-Duffing oscillator with asymmetric potential: chaotic behaviors predictions. Chinese Journal of Physics, 56, 1089-1104

Lam, L. (1997). Introduction to Nonlinear Physics. New York: Springer-Verlag.

Rand, R. H.(2005). Lecture Notes on Nonlinear Vibrations. Retrieved from http://audiophile.tam.cornell.edu/randdocs/nlvibe52.pdf

Nayfey, A. H., \& Mook, D. T. (1979).Nonlinear oscillations. New York: John Wiley and Sons.

\section{Copyrights}

Copyright for this article is retained by the author(s), with first publication rights granted to the journal.

This is an open-access article distributed under the terms and conditions of the Creative Commons Attribution license (http://creativecommons.org/licenses/by/4.0/). 